\title{
SATISFACCIÓN DE LOS ESTUDIANTES CON LA CALIDAD DE ENSEÑANZA DE LOS DOCENTES DE LA FACULTAD DE ENFERMERÍA DE LA UNIVERSIDAD NACIONAL "SAN LUIS GONZAGA" DE ICA, OCTUBRE 2013 - OCTUBRE 2014
}

\author{
Student satisfaction with the quality of education of teachers of Nursing Faculty of National \\ University "San Luis Gonzaga" of Ica, October 2013 - October 2014
}

\begin{abstract}
Isabel Natividad Urure Velazco ${ }^{1, a, e}$, Rosario Campos Soto ${ }^{2, c, d}$, Cecilia Teresa Ventura Miranda ${ }^{3, a, d}$, Olivia Hermelinda Helia Curasi Gómez, ${ }^{2, a, b}$, Lizeth Benny Gomez Acharte ${ }^{1, f}$
\end{abstract}

\footnotetext{
${ }^{1}$ Facultad de Enfermería. Universidad Nacional San Luis Gonzaga de Ica. Ica, Perú.

${ }^{2}$ Hospital Santa María del Socorro. Ica, Perú.

${ }^{3}$ Hospital Augusto Hernández Mendoza de Ica, Perú

${ }^{a}$ Lic. en Enfermería. ${ }^{b}$ Enfermera Especialista en Emergencias y Desastres, ${ }^{c}$ Mag. en Salud Pública . ${ }^{d}$ Mag. en Ciencias de la Enfermería, ${ }^{e}$ Doctorado en Ciencias de la Salud, ${ }^{\dagger}$ Estudiante de Enfermería
}

\section{RESUMEN}

La satisfacción del estudiante es descrita como elemento clave en la valoración de la calidad de la educación. Objetivo: Determinar la satisfacción de los estudiantes con la calidad de enseñanza de los Docentes de la Facultad de Enfermería de la Universidad Nacional "San Luis Gonzaga" de Ica. Material y métodos: Estudio descriptivo, transversal, diseño no experimental. Constituido por 143 estudiantes del I al X Ciclo. Se hizo uso de un cuestionario validado por expertos internacionales de 36 reactivos tipo escala Likert. Resultados: los estudiantes mayoritariamente manifestaron estar satisfechos con la calidad de enseñanza de los docentes $62,94 \%, 24,48 \%$ poco satisfecho, 4,90\% muy satisfecho, 4,20 insatisfechos y 3,50 muy insatisfechos. Con un promedio ponderado estimado de 4 en indicadores: Nivel de importancia y grado de satisfacción en aspectos: contenido de la unidad y grado de cumplimiento, metodología, desempeño del profesor y del estudiante, y 3 en infraestructura disponible. Conclusiones: Los estudiantes de la Facultad de Enfermería se sienten satisfechos con la calidad de enseñanza de los docentes con un promedio general estimado de 4. Con la contrastación de la hipótesis y aplicando la prueba estadística de T Student, se acepta la hipótesis de investigación (H1).

Palabras clave: Satisfacción, estudiantes, calidad, enseñanza, docente.

\section{SUMARY}

Student satisfaction is described as a key element in assessing the quality of education. Objective: To determine student satisfaction with the quality of teaching Teachers College of Nursing National "San Luis Gonzaga" of Ica University. Material and Methods: A descriptive, cross-sectional, nonexperimental design. Consisting of 143 students from I to $X$ Cycle. Use of a questionnaire validated by international experts from 36 reagents Likert scale was made. Results: Students overwhelmingly expressed satisfaction with the quality of teachers teaching $62,94 \%, 24,48 \%$ very satisfied, very satisfied 4, 90\%, 4, 20 and 3, 50 dissatisfied very dissatisfied. With a weighted average estimated 4 indicators: level of importance and satisfaction aspects: content of the drive and compliance, methodology, teacher performance and student, and 3 infrastructure available. Conclusions: Students in the School of Nursing are satisfied with the quality of teachers teaching with a general average of 4 With the testing of the hypothesis and applying the Student $t$ test statistic, the research hypothesis is accepted $(\mathrm{H} 1)$.

Keywords: Satisfaction, students, quality teaching, teacher. 


\section{INTRODUCCIÓN}

Uno de los grandes desafíos de la educación superior es sin duda, el mejoramiento de la calidad de los sistemas educativos, el tema de la calidad de la educación es un área prioritaria en nuestro medio en la actualidad. La universidad peruana, como todas en el mundo entero, ha atravesado por momentos difíciles y ha sido una crisis interna en la calidad de su formación, aunado a una serie de factores internos y externos en su conformación que han mellado esta crisis, sin embargo estos procesos de calidad que ya se desarrollan muchos años atrás en el mundo entero, resultan muy incipientes en el Perú introducir estos cambios en la educación superior, y por ende en las universidades significa instaurar procesos de acreditación calidad. La acreditación es el reconocimiento formal del cumplimiento de los estándares del modelo de calidad establecido para nuestra profesión (1).

En este sentido, según revisión bibliográfica señala el autor Arrien (2009) que dentro de un enfoque amplio y diverso de la calidad en la actualidad se considera la satisfacción que produce el proceso educativo en alumnos, profesores, padres y sociedad, un elemento consustancial de la calidad educativa (2). Por consiguiente, "la satisfacción del estudiante es descrita como elemento clave en la valoración de la calidad de la educación, se considera que uno de los indicadores más importantes para medir la calidad de la enseñanza tiene que ver con el grado de satisfacción de las personas involucradas en el proceso educativo" (3). El criterio de calidad de enseñanza reside en el grado en que los servicios ofertados satisfacen los requerimientos y expectativas de los estudiantes, un alumno satisfecho con la institución universitaria confirma la calidad de la misma, son los propios alumnos los que sostienen que estarán satisfechos en la medida en que los servicios que se les ofrecen responden a sus necesidades $y$ sucede cuando un producto o servicio cumple o excede las expectativas del cliente (4) (5). Es decir, en este estudio en particular lo constituyen los alumnos (clientes) de la Facultad de Enfermería estarán satisfechos si se cumplen en gran medida las expectativas de estos. "La actividad formativa de las facultades, se logra a través de los programas académicos, como de actividades de carácter multidimensional, en conjunto, ambos tipos de actividades constituyen un currículo integral al cual son expuestos los alumnos" (6). En consecuencia, se señala que en los últimos años la enseñanza universitaria ha sufrido cambios cuantitativos y cualitativos importantes en nuestro país (Perú), caracterizada por una demanda masiva de estudiantes y una mayor exigencia de la calidad de enseñanza, siendo uno de los enfoques que ha emprendido el sector universitario para hacer frente a los inminentes cambios que le exige la sociedad, es ofrecer los servicios educativos de calidad con la finalidad de cubrir las expectativas del estudiante. En este contexto, como antecedentes, se menciona a nivel internacional en España, estudios sobre satisfacción de los estudiantes con el proceso formativo año 2010, indican "en líneas generales como muy satisfechos" (7). Encontrándose similitud a nivel nacional Lima, Perú (2008) en estudiantes de Medicina de la Universidad Privada (8). No siendo consistente en Universidades Públicas donde manifestaron estar parcialmente satisfechos (8), y cifras o porcentajes aún menores $38,8 \%$ de satisfacción de los alumnos con el desempeño docente refieren en Lima, Perú año 2011 (9). Entonces se puede decir que, frente a la realidad que atraviesa la Facultad de Enfermería de la Universidad Nacional "San Luis Gonzaga" de Ica, en esta era neoliberal globalizada que exige competitividad y alcanzar estándares de calidad, existiendo brechas que aún todavía se observa en la calidad educativa, tratando de superarlos y llegar a la meta de ser una Facultad acreditada, es que nos motiva a indagar $y / 0$ investigar. Siendo asimismo 
importante este estudio realizado porque va a proporcionar datos a la Institución que permitirán mejorar la calidad de los sistemas educativos de los docentes de la Facultad de Enfermería, generando cambios, replanteando aspectos y superando las deficiencias encontradas, conducentes a la mayor satisfacción de los estudiantes universitarios. Señalándose como objetivo determinar la satisfacción de los estudiantes con la calidad de enseñanza de los datos de la Facultad de Enfermería de la Universidad Nacional "San Luis Gonzaga" de Ica.

\section{MATERIAL Y MÉTODOS.}

El estudio utilizó el método descriptivo, diseño no experimental de corte transversal, ejecutándose en la Facultad de Enfermería de la Universidad Nacional "San Luis Gonzaga" de Ica, ubicándose en la Ciudad Universitaria: Panamericana Sur Km. 305 Av. Los Maestros $\mathrm{S} / \mathrm{N}$ Ica, Perú, con una población de 530 estudiantes. Muestra constituida por 143 estudiantes del I al X Ciclo (o del $1^{\circ}$ Año al $5^{\circ}$ Año). Se obtuvo este tamaño muestral con una fórmula para población finita. Se excluyeron estudiantes que estuvieron realizando prácticas pre-profesionales (internado) fuera de la ciudad de Ica.

Haciendo uso de un cuestionario que estuvo estructurado de 4 partes: Primer parte: Relacionado a datos sociodemográficos: Sexo, edad, estado civil, año de estudios, ocupación de padres, lugar de residencia, medio de transporte, accesibilidad a la Facultad. Segunda parte: Referente a importancia de aspectos que influyen en la satisfacción del estudiantes, con 5 reactivos medidos con una escala Likert. Referente a premisas: contenido de la unidad de aprendizaje, metodología, infraestructura, desempeño del profesor y estudiante. Tercer parte: Con reactivos que midieron el desempeño de enseñanza de los docentes, utilizándose 30 reactivos tipo escala Likert con una valoración del 1 al 5 para muy insatisfecho, insatisfecho, poco satisfecho o medianamente satisfecho, satisfecho y muy satisfecho respectivamente, relativos a: Contenido de la unidad de aprendizaje y grado de cumplimiento, metodología con 5 reactivos cada uno, infraestructura 3 reactivos, desempeño del profesor y del estudiante con 10 y 7 reactivos respectivamente. Cuarta parte: Satisfacción global con 1 reactivo de valoración cualitativa, con 5 niveles: Muy insatisfecho, insatisfecho, poco satisfecho, satisfecho y muy satisfecho (similar a la anterior). Se utilizó un total de 36 reactivos.

En la recolección de datos se empleó como técnica la encuesta y como instrumento se utilizó un cuestionario de Jiménez $A$. et al. que fue validado por expertos internacionales y aplicada a trabajos de investigación sobre satisfacción de estudiantes en México.

Se hizo uso del programa de análisis estadístico de datos SPSS Versión 21.

Para prueba de hipótesis se aplicaron las pruebas de U-MANN-WHITNEY para relación entre variables ordinales - nominales dicotómicas, el coeficiente de correlación Rho-Spearman para la asociación entre variables ordinales y la prueba de T-KruskalWallis para la contrastación entre variables nominales politómicas. Para la contrastación de hipótesis se utilizó la prueba de T Student.

\section{RESULTADOS.}

El promedio ponderado de la importancia de los 5 aspectos que influyen en la satisfacción del estudiante fue de 3,97. Respecto al desempeño en indicadores: Contenido de la unidad y grado de cumplimiento fue de 3,54, metodología 3,66, infraestructura disponible 3,23 , desempeño del profesor y estudiante 3,70 y 3,88 respectivamente. El promedio ponderado general estimado fue de 4 puntos en un rango de puntuación de 1 a 5 , lo que corresponde a la categoría de Satisfacción de la variable principal estudiada; esto nos permite concluir que los alumnos de la Facultad de Enfermería de la UNICA en el 
periodo de estudios perciben como satisfactoria con la calidad de enseñanza de los docentes. (Tabla 1)

Respecto al grado de satisfacción global fue predominante satisfecho con 62,94\% (90 estudiantes) poco satisfecho 24,48\% (35 estudiantes), muy satisfecho 4,20\% (6 estudiantes) y con un nivel de insatisfacción $7,7 \%$ (11 estudiantes) (Tabla 2). La relación entre las variables sociodemográficas con el grado de satisfacción global: Las mayores frecuencias se presentaron en el nivel de satisfecho asociándose esta última con el sexo femenino 55,24\% (79 estudiantes), edad 20-24 años 37,60\% (53 estudiantes), primero a quinto año $14,69 \%$ (21 estudiantes), el tener padres de ocupación trabajador independiente 20,18\% (29 estudiantes), residir en área urbana $53,15 \%$ (76 estudiantes), emplear vehículos como medio de transporte $44,76 \%$ (64 estudiantes) y emplear de 11 a 20 minutos para desplazarse a la universidad $23,78 \%$ (34 estudiantes). Como prueba de hipótesis se aplicaron las pruebas de U-MANN-WHITNEY para relación entre variables ordinalesnominales dicotómicas, el coeficiente de correlación de Rho-Spearman para asociación entre variables ordinales, y TKruskal-Wallis para contrastación entre variables ordinales-nominales politómicas, resultando en todos los casos no significativo a un $p-$ valor $\leq 0,05$.(Tabla 3 ).

\section{TABLA № 1: PROMEDIO PONDERADO DE LA SATISFACCIÓN DE LOS ESTUDIANTES CON LA CALIDAD DE ENSEÑANZA DE LOS DOCENTES DE LA FACULTAD DE ENFERMERÍA DE LA UNICA.}

\begin{tabular}{lc}
\hline \multicolumn{1}{c}{ Satisfacción } & $\begin{array}{c}\text { Promedio } \\
\text { ponderado }\end{array}$ \\
\hline IMPORTANCIA & 3,97 \\
\hline Aspectos que influyen en la satisfacción del estudiante & \\
\hline DESEMPEÑO & 3,54 \\
Contenido de la Unidad y Grado de Cumplimiento & 3,66 \\
Metodología & 3,23 \\
Infraestructura disponible & 3,70 \\
Desempeño del profesor & 3,88 \\
\hline Mi desempeño & 3,66 \\
\hline Promedio General & \\
\hline
\end{tabular}

Fuente: Base de datos

TABLA N 02: DISTRIBUCIÓN DE LOS ALUMNOS ENCUESTADOS SEGÚN EL GRADO DE SATISFACCIÓN GLOBAL CON LA CALIDAD DE ENSEÑANZA EN LA FACULTAD DE ENFERMERÍA DE LA UNICA.

\begin{tabular}{|c|c|c|c|c|c|c|c|c|c|c|}
\hline \multirow{3}{*}{ Premisa } & \multicolumn{10}{|c|}{$N=143$} \\
\hline & \multicolumn{2}{|c|}{$\begin{array}{c}\text { Muy } \\
\text { insatisfecho }\end{array}$} & \multicolumn{2}{|c|}{ Insatisfecho } & \multicolumn{2}{|c|}{$\begin{array}{c}\text { Poco } \\
\text { satisfecho }\end{array}$} & \multicolumn{2}{|c|}{ Satisfecho } & \multicolumn{2}{|c|}{$\begin{array}{c}\text { Muy } \\
\text { satisfecho }\end{array}$} \\
\hline & $n$ & $\%$ & $\mathrm{n}$ & $\%$ & $\mathrm{n}$ & $\%$ & $\mathrm{n}$ & $\%$ & $n$ & $\%$ \\
\hline $\begin{array}{l}\text { En términos generales, } \\
\text { ¿cómo se siente con la } \\
\text { calidad de enseñanza que } \\
\text { le brindan los docentes?. }\end{array}$ & 5 & 3,50 & 6 & 4,20 & 35 & 24,48 & 90 & 62,94 & 7 & 4,90 \\
\hline
\end{tabular}


TABLA N` 03: RELACIÓN ENTRE VARIABLES SOCIO DEMOGRÁFICAS Y GRADO DE SATISFACCIÓN GLOBAL DE LOS ESTUDIANTES CON LA CALIDAD DE ENSEÑANZA EN LA FACULTAD DE ENFERMERÍA DE LA UNICA

\begin{tabular}{|c|c|c|c|c|c|c|c|c|c|c|c|}
\hline \multirow{4}{*}{$\begin{array}{l}\text { Variables } \\
\text { Sexo }\end{array}$} & \multicolumn{10}{|c|}{ Grado de Satisfacción Global } & \multirow{3}{*}{$\begin{array}{c}\text { Prueba de } \\
\text { Hipótesis }\end{array}$} \\
\hline & \multicolumn{10}{|c|}{$N=143$} & \\
\hline & \multicolumn{2}{|c|}{$\begin{array}{c}\text { Muy } \\
\text { insatisfecho }\end{array}$} & \multicolumn{2}{|c|}{ Insatisfecho } & \multicolumn{2}{|c|}{$\begin{array}{c}\text { Poco } \\
\text { satisfecho }\end{array}$} & \multicolumn{2}{|c|}{ Satisfecho } & \multicolumn{2}{|c|}{$\begin{array}{c}\text { Muy } \\
\text { satisfecho }\end{array}$} & \\
\hline & $\mathrm{n}$ & $\%$ & $n$ & $\%$ & $\mathrm{n}$ & $\%$ & $\mathrm{n}$ & $\%$ & $\mathrm{n}$ & $\%$ & $p$ - valor \\
\hline Femenino & 5 & 3,50 & 5 & 3,50 & 27 & 18,88 & 79 & 55,24 & 6 & 4,20 & $0,406^{*}$ \\
\hline Masculino & 0 & 0,00 & 1 & 0,70 & 8 & 5,59 & 11 & 7,69 & 1 & 0,70 & \\
\hline Grupo etario & $\mathrm{n}$ & $\%$ & $\mathrm{n}$ & $\%$ & $\mathrm{n}$ & $\%$ & $\mathrm{n}$ & $\%$ & $n$ & $\%$ & $\mathrm{p}$ - valor \\
\hline $15-19$ & 1 & 0,70 & 2 & 1,40 & 10 & 6,99 & 36 & 25,17 & 3 & 2,10 & $0,163^{\star *}$ \\
\hline $20-24$ & 4 & 2,80 & 4 & 2,80 & 22 & 15,38 & 53 & 37,06 & 3 & 2,10 & \\
\hline $25-29$ & 0 & 0,00 & 0 & 0,00 & 3 & 2,10 & 1 & 0,70 & 0 & 0,00 & \\
\hline $30-34$ & 0 & 0,00 & 0 & 0,00 & 0 & 0,00 & 0 & 0,00 & 1 & 0,70 & \\
\hline Año de estudios & $\mathrm{n}$ & $\%$ & $n$ & $\%$ & $n$ & $\%$ & $n$ & $\%$ & $n$ & $\%$ & $p$ - valor \\
\hline Primero & 1 & 0,70 & 1 & 0,70 & 5 & 3,50 & 21 & 14,69 & 3 & 2,10 & $0,444^{\star \star}$ \\
\hline Segundo & 0 & 0,00 & 1 & 0,70 & 9 & 6,29 & 19 & 13,29 & 0 & 0,00 & \\
\hline Tercero & 2 & 1,40 & 2 & 1,40 & 6 & 4,20 & 16 & 11,19 & 1 & 0,70 & \\
\hline Cuarto & 1 & 0,70 & 0 & 0,00 & 11 & 7,69 & 13 & 9,09 & 3 & 2,10 & \\
\hline Quinto & 1 & 0,70 & 2 & 1,40 & 4 & 2,80 & 21 & 14,69 & 0 & 0,00 & \\
\hline Ocupación de padres & $\mathrm{n}$ & $\%$ & $\mathrm{n}$ & $\%$ & $\mathrm{n}$ & $\%$ & $\mathrm{n}$ & $\%$ & $\mathrm{n}$ & $\%$ & $p$ - valor \\
\hline Ama de casa & 0 & 0,00 & 0 & 0,00 & 2 & 1,40 & 4 & 2,80 & 0 & 0,00 & $0,908^{\star \star \star}$ \\
\hline Estudiante & 1 & 0,70 & 0 & 0,00 & 6 & 4,20 & 10 & 6,99 & 0 & 0,00 & \\
\hline Empleado & 1 & 0,70 & 2 & 1,40 & 9 & 6,29 & 26 & 18,18 & 4 & 2,80 & \\
\hline $\begin{array}{l}\text { Trabajador } \\
\text { independiente }\end{array}$ & 1 & 0,70 & 2 & 1,40 & 10 & 6,99 & 29 & 20,28 & 1 & 0,70 & \\
\hline Obrero & 0 & 0,00 & 1 & 0,70 & 2 & 1,40 & 6 & 4,20 & 1 & 0,70 & \\
\hline Agricultor/Ganadero & 1 & 0,70 & 0 & 0,00 & 0 & 0,00 & 4 & 2,80 & 0 & 0,00 & \\
\hline Otros & 1 & 0,70 & 1 & 0,70 & 6 & 4,20 & 11 & 7,69 & 1 & 0,70 & \\
\hline Lugar de residencia & $\mathrm{n}$ & $\%$ & $\mathrm{n}$ & $\%$ & $\mathrm{n}$ & $\%$ & $\mathrm{n}$ & $\%$ & $n$ & $\%$ & $p$ - valor \\
\hline Urbano & 4 & 2,80 & 6 & 4,20 & 30 & 20,98 & 76 & 53,15 & 6 & 4,20 & $0,705^{\star}$ \\
\hline Urbano - marginal & 1 & 0,70 & 0 & 0,00 & 5 & 3,50 & 14 & 9,79 & 1 & 0,70 & \\
\hline Medio de transporte & $\mathrm{n}$ & $\%$ & $n$ & $\%$ & $\mathrm{n}$ & $\%$ & $\mathrm{n}$ & $\%$ & $\mathrm{n}$ & $\%$ & $p$ - valor \\
\hline Vehículo & 5 & 3,50 & 3 & 2,10 & 24 & 16,78 & 64 & 44,76 & 5 & 3,50 & $0,847^{\star \star \star}$ \\
\hline A pie & 0 & 0,00 & 1 & 0,70 & 8 & 5,59 & 17 & 11,89 & 0 & 0,00 & \\
\hline Otros & 0 & 0,00 & 2 & 1,40 & 3 & 2,10 & 9 & 6,29 & 2 & 1,40 & \\
\hline $\begin{array}{l}\text { Accesibilidad } \\
\text { (minutos) }\end{array}$ & $\mathrm{n}$ & $\%$ & $n$ & $\%$ & $\mathrm{n}$ & $\%$ & $\mathrm{n}$ & $\%$ & $n$ & $\%$ & $p$ - valor \\
\hline$\leq 10$ & 1 & 0,70 & 3 & 2,10 & 9 & 6,29 & 26 & 18,18 & 1 & 0,70 & $0,619^{\star *}$ \\
\hline $11-20$ & 0 & 0,00 & 1 & 0,70 & 11 & 7,69 & 34 & 23,78 & 2 & 1,40 & \\
\hline $21-30$ & 3 & 2,10 & 2 & 1,40 & 6 & 4,20 & 16 & 11,19 & 3 & 2,10 & \\
\hline $31-40$ & 0 & 0,00 & 0 & 0,00 & 4 & 2,80 & 10 & 6,99 & 0 & 0,00 & \\
\hline $41-50$ & 1 & 0,70 & 0 & 0,00 & 3 & 2,10 & 4 & 2,80 & 0 & 0,00 & \\
\hline $51-60$ & 0 & 0,00 & 0 & 0,00 & 2 & 1,40 & 0 & 0,00 & 1 & 0,70 & \\
\hline
\end{tabular}

Fuente: Base de datos.

*Prueba U-Mann Whitney

**Prueba Rho- Spearman

***Prueba T-Kruskal Wallis 


\section{DISCUSIÓN.}

La satisfacción de los estudiantes con la calidad de enseñanza de los docentes respecto al indicador importancia fue buena con $70 \%$ y con un promedio ponderado de 3,97 de un puntaje de 1 a 5 que equivale al nivel de satisfecho, existiendo todavía una brecha en promedio de 1,05 para alcanzar el nivel óptimo de importancia que dan los estudiantes en los 5 aspectos consideraqdos que influyen en la satisfacción. Este hallazgo es consistente con Jiménez $A$, et al. En México, cuyo resultado fue $71 \%$ (10). Referente al desempeño en sus diversos aspectos, el estudio mostró como el aspecto mejor evaluado al desempeño del estudiante con un promedio ponderado de 3,88, encontrándose coincidencia con el hallazgo de Jiménez $A$, et al. con $68 \%$ señalando también al estudiante como el aspecto mejor evaluado (10). En este sentido, se evidencia cifras favorables de consideración respecto a la satisfacción del desempeño de los estudiantes, siendo factor predisponente mayoritario la motivación de clases, lo interesante y novedoso de ellas, que influyeron en el cumplimiento de tareas, etc, pero todavía hay un promedio de 1,12 por trabajar para alcanzar el puntaje óptimo de 5 con tutoría personalizada para mejorar el desempeño continuo del estudiante.

En lo concerniente al desempeño de los demás aspectos, los promedios ponderados fueron casi semejantes con una ligera diferencia entre desempeño del profesor 3,70 y metodología 3,66, mostrando el estudio similitud con el hallazgo de Jiménez $A$ et al. respecto al desempeño del profesor con un $64 \%$ (10). Corimayhua en Perú señalan cifras un tanto inferior, siendo, el resultado de una relación directa entre el desempeño profesional docente y la satisfacción de los alumnos en un $38,8 \%$ (9). No mostrando consistencia el aspecto de metodología con el nuestro que ocupó el tercer lugar en secuencia con Jiménez $A$. et al. quien ubica a metodología en cuarto lugar con 53\% (10). De lo expuesto, en el estudio consideramos que los estudiantes mostraron satisfacción en resultados casi similares entre el desempeño del docente y la metodología empleada, aspectos importantes que cubren mayoritariamente las expectativas del alumno que le estimula a adquirir nuevos conocimientos y afianzar los ya adquiridos. Sin embargo existe todavía promedios porcentuales de 1,30 y 1,34 de brecha que no mostraron satisfacción, y se tiene que seguir monitorizando y mejorar en la dinámica grupal, promover la discusión, el diálogo, análisis crítico y lectura veloz en los estudiantes y alcanzar estándares de calidad de enseñanza - aprendizaje.

La unidad de aprendizaje en las 5 premisas alcanzó el promedio ponderado de 3,54, respondiendo mayoritariamente en el contenido de la unidad y objetivo del mismo, existiendo todavía un $43 \%$ que no mostraron satisfacción, permitiendo estos resultados controlar y establecer nuevas estrategias en ítems que no señalaron satisfacción, como el tiempo de duración de la unidad de aprendizaje, etc, y alcanzar la meta trazada, conducente a mejorar la calidad de aprendizaje, propiciando en el estudiante a adquirir un espíritu crítico, analítico e innovador, que es lo que requiere la sociedad actual de competitividad. El estudio no mostró consistencia con el de Jiménez $A$ et al, cuyo resultado fue de $62 \%$, ubicándole en tercer lugar, a diferencia de nuestro estudio que ocupó el cuarto lugar en ese rubro. En infraestructura, la muestra encontró un promedio ponderado inferior de 3,23 en sus tres premisas, coincidente con estudios de Corimayhua en Lima, Perú (9), y Jiménez A et al. (10), con cifras casi similares $36,9 \%$ y $41 \%$ respectivamente, considerando la relación directa entre la infraestructura y la satisfacción de los alumnos, siendo un elemento tangible de importancia y decisorio para resultados positivos de satisfacción.

La relación entre las variables sociodemográficas y grado de satisfacción global, se aplicaron pruebas de hipótesis UMANN-WHITNEY, Coeficiente de Correlación de RHO-SPEARMAN, y prueba de TKRUSKAL WALLIS, resultando en todos los casos no significativas en $p-$ valor $\leq 0,05$. No encontrándose consistencia estos resultados 
con el estudio de Tantas G, en Lima, Perú año 2008. que demostraron que las variables sociodemográficas influyen en la calidad de enseñanza (8).

El grado de satisfacción global de los estudiantes con la calidad de enseñanza de los docentes fue de satisfecho con $62,94 \%$, contrastando con antecedentes se observó resultados inferiores de satisfacción con el estudio de Jiménez $A$ et al. en donde los autores concluyen estar por arriba de $58 \%$ de satisfacción (10). Cifras aún menores señala Corimayhua en Lima, Perú, mostrando que existe una relación significativa entre la calidad del servicio educativo y la satisfacción de los alumnos de la ciudad de Lima, en un $53,62 \%$ (9). Asimismo se puede señalar en nuestro estudio, indicadores como importancia y desempeño en sus aspectos (unidad de aprendizaje, metodología, infraestructura, desempeño del profesor y estudiante) se obtuvo como promedio general estimado 4 de un puntaje del 1 al 5, correspondiendo al nivel de satisfecho, coincidente con el hallazgo porcentual obtenido en el estudio.

\section{CONCLUSIONES.}

Los alumnos de la facultad de enfermería de la Universidad Nacional "San Luis Gonzaga "de Ica, se sienten satisfechos con la calidad de enseñanza de los docentes, con un porcentaje de $62,94 \%$, con un promedio ponderado general estimado de 4 de un puntaje del 1 al 5 . Se contrasta la hipótesis y se aplicó la prueba estadística de $\mathrm{T}$ de Student, aceptándose la hipótesis de investigación $(\mathrm{H} 1)$. Existiendo todavía un porcentaje y promedio global de diferencia para alcanzar el nivel óptimo de satisfacción con la calidad educativa de 37,06 y 1 respectivamente

\section{RECOMENDACIONES:}

Para alcanzar niveles óptimos de satisfacción y superar brechas existentes, se debe implementar planes de mejora continua a fin de eliminar debilidades encontradas $y$ potencializar las existentes.

\section{Correspondencia:}

Dra. Isabel N. Urure Velazco.

Correo electrónico: isabelnauv@yahoo.com

\section{REFERENCIAS BIBLIOGRÁFICAS}

1. Pecho M. Hacia la acreditación. Facultad de Enfermería. Universidad Nacional "San Luis Gonzaga” de Ica. Ica, Perú. 2011

2. Arríen J. La satisfacción, ¿elementos de la Calidad educativa?. El nuevo diario. Noriega, Nicaragua. 2009. Citado el 07 de Agosto 2013. Disponible en: http://impreso.elnuevodi ario.comni/2009/08/07/ opinión/

3. Zas, B. La satisfacción como indicador de excelencia en la calidad de los servicios de salud. Publicado en la revista electrónica psicología científica. 2002. Citado Setiembre 2013. Disponible en: http://psicologia cientifica.com

4. Liljander, V. Modeling perceived service quality using different comparison standards. Journal of Consumer Satisfaction, Dissatisfaction and Complaining Behaviour, 1994. 7, 126-142.

5. Dabholkar, P. A contingency framework for predicting causality between customer satisfaction and service quality. Advances in Consumer Research. 1995. 22, 101-108

6. Casassus, J. La escuela y la desigualdad. LOM Ediciones. 1era ed., Santiago de Chile. 2003.

7. Lepiani I, Dueñas M, Medialdera M. J., Bocchino A. Satisfacción de Estudiantes de Enfermería con el proceso formativo adaptado al espacio europeo de Educación Superior. Tesis. Universidad de Cádiz. Andalucía, España. 2010. 
8. Tantas G. "Evaluación De Calidad De Enseñanza Desde La Perspectiva De Satisfacción De Los Estudiantes De Medicina De La Universidad Nacional Federico Villarreal $Y$ Universidad Privada Ricardo Palma, Lima - Perú, 2008" Tesis Magistral. Lima, Perú. Facultad de Medicina. Universidad Nacional Federico Villareal. 2008.

9. Corimayhua A. "Calidad De Los Servicios Educativos $Y$ La Satisfacción De Los Alumnos De Los Colegios Adventistas De Lima". Tesis. Universidad Peruana Unión. Lima, Perú. 2011.
10. Jiménez A, Terriquez $B$, Robles $\mathbf{F}$. "Evaluación de la Satisfacción Académica de Los Estudiantes de la Universidad Autónoma de Nayarit" Tesis. México 2011.

Recibido: 01/10/2014

Aprobado para publicación: 01/12/2014 